\title{
Variations sur un dessin à la mine de plomb «Progression dans un paysage enneigé » en regard des Géorgiques
}

Pierre Schoentjes et Griet Theeten

\section{(2) OpenEdition \\ Journals}

Édition électronique

URL : http://journals.openedition.org/ccs/818

DOI : $10.4000 /$ ccs. 818

ISSN : 2558-782X

Éditeur :

Presses universitaires de Rennes, Association des lecteurs de Claude Simon

Édition imprimée

Date de publication : 31 décembre 2011

Pagination : 61-78

ISBN : 9782354121464

ISSN : $1774-9425$

Référence électronique

Pierre Schoentjes et Griet Theeten, « Variations sur un dessin à la mine de plomb « Progression dans un paysage enneigé » en regard des Géorgiques », Cahiers Claude Simon [En ligne], 7 | 2011, mis en ligne le 21 septembre 2017, consulté le 02 mai 2019. URL : http://journals.openedition.org/ccs/818 ; DOI : $10.4000 /$ ccs. 818 


\title{
Variations sur un dessin à la mine de plomb "Progression dans un paysage enneigé " en regard des Géorgiques
}

\author{
Pierre SCHOENTJES \& Griet THEETEN \\ Université de Gand
}

Lorsqu'il est en train de rédiger Les Géorgiques (1981), Claude Simon publie dans Études littéraires quelques extraits de ce travail en cours : "Progression dans un paysage enneigé » (1976). Il y met en scène un cavalier qui parcourt la Flandre enneigée pendant un hiver que nous pouvons identifier comme l'hiver de 1939-1940. La scène est particulièrement visuelle et évoque les "dessins minutieux et précis à la mine de plomb ${ }^{1}$ chers à l'auteur. Le titre, " progression ", désigne en premier lieu le mouvement qui caractérise les trois sections de ce texte. En effet, tout est constamment en mouvement ou semble l'être. Mais une deuxième signification s'y ajoute : les trois fragments de "Progression dans un paysage enneigé » ne constituent pas des scènes qui se font suite. Au contraire, il s'agit de trois réécritures d'une même expérience, chacune portant l'accent sur de nouveaux éléments. Nous pouvons donc interpréter le titre

1. Claude Simon, Les Géorgiques, Paris, Minuit, 1981, p. 85 (dorénavant abrégé comme $G$ ). 
comme une suite de variations, comme dans la forme musicale de la fugue.

Ce court texte nous invite ainsi à examiner de près sa structure, son fonctionnement interne et les glissements de sens concomitants. L'angle d'approche par lequel nous avons choisi d'étudier ces phénomènes est double. Dans un premier temps, nous nous arrêterons à l'écriture sensorielle qui caractérise "Progression " en analysant les champs lexicaux, métaphores et motifs liés aux différents sens. En deuxième lieu, nous regarderons l'évolution dans le style des trois sections constituant "Progression ». Une analyse des comparaisons, des métaphores mais aussi du rythme s'impose ici puisque ce texte illustre les jeux de variations - au sens musical du terme - à l'œuvre dans la production de Claude Simon.

Si «Progression dans un paysage enneigé " mérite d'être analysé comme un texte isolé, il nous intéresse tout autant pour la relation intratextuelle qu'il entretient avec Les Géorgiques, livre auquel Simon a travaillé simultanément. Les impressions de lecture évoquées par la lente progression du cavalier dans le texte court rejoignent celles que suscitent les pages des Géorgiques où un groupe de cavaliers marche dans une séquence de guerre ${ }^{2}$. Dès lors, il est possible d'effectuer une lecture serrée qui rende compte des jeux de variations qui s'opèrent entre « Progression » et le roman ultérieur.

\section{Une écriture sensorielle}

Dans "Progression dans un paysage enneigé ", Claude Simon s'efforce de rendre compte des sensations les plus infimes, qui prennent forme à partir de notations précises. La description minutieuse

2. Le "il " de " Progression dans un paysage enneigé ", une troisième personne du singulier non identifiée, deviendra un « il » identifiable dans le chapitre II des Géorgiques, dans lequel on reconnaît le cavalier de la Seconde Guerre mondiale. Rappelons toutefois que, dans le premier et le dernier chapitre du roman, le pronom « il » indique tour à tour le cavalier, O., L.S.M. ou l'écrivain, créant ainsi chez le lecteur un sentiment de confusion. Les traces de "Progression " se retrouvent essentiellement dans ce deuxième chapitre, mais nous en trouvons également dans le premier et le dernier chapitre du roman $(G$ p. 90, 97, 102, 104, 108, 118, 119, $121,128,134,135,422)$. 
des bruits de la nature, du ciel d'hiver et du paysage environnant le cavalier, cette attention au détail justifie à elle seule qu'on s'intéresse à ce texte. Chaque section semble développer d'autres images et apporter des motifs inédits. L'expression de la sensation du froid illustre bien ce mode de développement. L'auteur mentionne déjà dans le premier extrait la neige qui recouvre le paysage mais ce n'est qu'à partir de la deuxième section qu'il insiste sur les températures extrêmes qui font souffrir le cavalier : «Malgré la protection du gant un étau douloureux écrase l'extrémité des doigts de la main qui tient les rênes » $(S 2, \text { p. 218 })^{3}$. Après les mains de son personnage, l'auteur s'attarde sur les pieds douloureux : « Dans les bottes durcies par le gel, les pieds semblent faire bloc avec le cuir » (S2, p. 218). La dernière section accumule encore les références à un froid hors du commun. La sensation de pieds gelés, qui n'occupe qu'une seule phrase dans la deuxième section, s'étale sur plusieurs phrases dans la troisième, comme si la voix narrative entendait souligner l'idée de souffrance :

Le froid enserre ses pieds dans des bottes de fer. Il peut le sentir gagner lentement de proche en proche et remonter le long de ses jambes. (S3, p. 219)

Il lui semble qu'un clou de glace est enfoncé sous chacun des ongles de la main qu'il élève maintenant à hauteur de sa poitrine [...]. (S3, p. 219-220)

Ses pieds lui semblent pris dans des étriers de froid. (S3, p. 220).

L'analyse des impressions auditives révèle un jeu sur le silence et le bruit qui se déploie au fur et à mesure du déroulé du texte. Parmi les rares sons décrits, Simon prête une attention particulière à celui produit par le métal : les mouvements du cheval font sonner le sabre, les étriers, le mors et les filets :

Les seuls bruits que l'on entende sont ceux du souffle de l'animal, parfois coupé par une expiration plus violente comme un éternuement, un tintement d'acier (le mors et le filet mâchonnés, le fourreau du sabre

3. Claude Simon, "Progression dans un paysage enneigé ", Études littéraires, IX, 9 , avril 1976, p. 217-219, abrégé dorénavant comme $P$. Nous ferons référence aux trois sections de ce texte par les abréviations S1, S2 et S3. 
contre l'étrier) ou le craquement assourdi d'une branche morte que la jument écrase au passage. (S1, p. 217)

Secoués par les efforts vigoureux de l'animal, le fourreau du sabre et un étrier s'entrechoquent avec un tintement clair dans le silence qui l'absorbe aussitôt. (S2, p. 218)

Le fourreau de son sabre tinte à chaque foulée du cheval contre l'étrier droit et avec le souffle de la bête c'est le seul bruit que l'on entende, insolite, énorme, dans la chape de silence. (S3, p. 220)

À l'exception du son du métal, tous les autres bruits s'amplifient à mesure qu'on avance dans le texte. Ainsi les paquets de neige qui tombent des arbres, interrompant le silence complet. Ce bruit demeure quasiment inaudible dans la première section du texte : "Parfois le déplacement de l'air, ou peut-être les imperceptibles vibrations du sol frappé par les sabots, font se détacher d'une branche un paquet de neige qui glisse lui aussi sans bruit » (S1, p. 217). Dans la deuxième section, Simon s'attarde sur le parcours que décrivent les paquets de neige, sans s'arrêter au bruit ou à l'absence de bruit qui le marque :

Parfois, sans raison apparente, sans que rien n'ait bougé dans les taillis, sans qu'il ait vu s'envoler un oiseau, des paquets de neige se détachent, glissent de rameau en rameau, entraînant d'autres paquets en une petite avalanche qui se pulvérise sur une branche basse [...] (S2, p. 218)

Dans la troisième section enfin, Claude Simon combine informations sur le son et le mouvement, en comparant la neige qui tombe à une cascade et à un rideau blanc. Le bruit généré devient ici audible :

Il arrive aussi que lorsqu'ils passent sous une branche lourdement chargée, les trépidations pourtant imperceptibles du sol, ou encore le déplacement de l'air, déclenchent la chute d'un gros paquet de neige qui tombe avec un faible chuintement, comme une cascade, un rideau blanc, juste après leur passage, s'affalant sur le sol avec un bruit mou, poudrant quelquefois la croupe du cheval sur laquelle les cristaux scintillent un instant avant de s'éteindre ${ }^{4}$. (S3, p. 220-221)

4. Nous soulignons. 
La même évolution du silence total vers la présence d'un léger bruit se vérifie dans la description des sabots qui frappent le sol. Les sabots qui, dans un premier temps, "s'enfoncent en silence dans la neige " $(\mathrm{S} 1, \mathrm{p} .217)$, se heurtent dans la deuxième section à une branche morte invisible sous la neige que l'" on entend parfois craquer avec un bruit assourdi » (S2, p. 219). Dans la troisième section, enfin, le bruit s'intensifie : "Pendant quelques instants il continue au trot et il peut maintenant entendre la mince couche de glace qui craque sous les sabots»(S3, p. 221).

À ce jeu subtil sur le bruit et le silence, Claude Simon ajoute l'opposition entre le mouvement et l'inaction : l'auteur ne cesse d'apporter des détails neufs sur les actions du cheval et du cavalier. Alors que dans la première section le cheval "se faufile entre les troncs noirs des arbres" (S1, p. 217) - la phrase crée une impression de lenteur -, les arbres défilant aux côtés du cavalier dans la deuxième section suggèrent une vitesse de déplacement plus élevée :

Autour de lui les troncs verticaux passent les uns derrière les autres comme les barreaux de grilles animées d'un lent mouvement horizontal, à des vitesses qui varient selon la distance 5 . (S2, p. 218)

Pareillement, la démarche du cheval n'est pas décrite dans la première section alors que les deux autres s'arrêtent longuement sur les mouvements spécifiques de l'animal :

Parfois la neige accumulée force la jument à ralentir et elle n'avance plus qu'au pas, levant haut ses jambes de devant, comme si elle piaffait, dégageant son arrière-train par de petites croupades et laissant derrière elle dans la neige un profond sillon coupé d'éboulis. Son souffle alors s'accélère. [...] La jument donne de musculeux coups de reins pour se dégager des congères. (S2, p. 218)

Bientôt une légère vapeur s'élève autour du poitrail et des épaules du cheval qui parfois ralentit, prend le pas et peine pour se dégager d'une congère, arrachant ses jambes l'une après l'autre, balançant son encolure et s'aidant de coups de reins nerveux. (S3, p. 219)

5. Observons que Simon note qu'à cause de la vitesse prise la neige, tombant des arbres " plaque sur son visage une poudre de paillettes » $(\mathrm{S} 2, P, 219)$. 
En outre, plus le cheval semble bouger, plus nous voyons s'animer le cavalier. La mobilité du personnage demeure restreinte dans la première section : "Sans presque qu'il ait à la guider sa monture se faufile entre les troncs noirs des arbres. De temps en temps il est obligé de se pencher sur l'encolure pour éviter une branche basse " (S1, p. 217). Simon souligne encore la quasi-inaction du cavalier au début de la deuxième section : "C'est tout juste s'il a besoin de déplacer légèrement sa main pour infléchir la course de la jument " (S2, p. 218), pour mentionner ensuite un semblant d'action : "Il l'aide à prendre appui sur le mors en élevant la main qui tient les rênes et serre les cuisses pour se maintenir en selle " (S2, p. 218). Dans la troisième section, en revanche, le cavalier devient nettement plus actif. Le narrateur détaille les efforts qu'il déploie pour garder le contrôle du cheval et pour éviter les branches des arbres ${ }^{6}$.

[... le cavalier,] obligé toutefois de serrer les genoux et de se pencher en arrière pour amortir les soubresauts du grand corps musculeux. (S3, p. 219)

Souvent il est obligé d'incliner son buste en avant ou sur le côté pour éviter d'être griffé par une branche basse ou passer au-dessous, ou encore il sort sa main gauche de sous son manteau pour écarter un rameau. (S3, p. 220) p. 221)

À la fin il serre un peu les jambes et met le cheval au galop. (S3,

Malgré la prédominance du mouvement et de l'action, le jeu de contraste entre mobilité et immobilité ne disparaît pas entièrement dans la troisième section. La comparaison entre la couche de neige et une mer gelée vers la fin de "Progression dans un paysage enneigé " en constitue l'exemple le plus significatif :

Par endroits les effets du vent, les tourbillons entre les arbres, ont modelé la neige en forme de vagues et sous les épaisses crêtes d'écume solidifiée on peut voir les herbes courbées par leur poids, solidifiées aussi, gelées et jaunies, nécrosées. (S3, p. 220)

6. L'écrivain renoue avec la figure du centaure qui lui est familière depuis La Route des Flandres (Paris, Minuit, 1960, p. 52-53) et qui rejoint les êtres hybrides peuplant Les Géorgiques: l'homme-pingouin ( $G$, p. 155), l'homme-babouin ( $G$, p. 152-153)... 
Prolifération manifeste des détails sensoriels, donc, que donne à lire Claude Simon dans "Progression »: du paysage enneigé vers le froid douloureux, du silence vers le bruit feutré, de l'inaction vers l'action. Il y a toutefois une constante dans les trois sections du texte court : que l'auteur s'attache à la nature, au personnage ou au cheval, à l'odorat, l'ouïe, la vue ou le goût, c'est toujours le champ lexical du métal qui prédomine. Pour peindre la couleur du ciel, par exemple, le romancier fait constamment référence au fer ou au cuivre ${ }^{7}$. Les paillettes de neige ont une saveur de métal, " comme des particules de fer " (S3, p. 220) quand elles touchent la bouche. Il serait ainsi possible de multiplier les exemples. Dans le contexte d'une promenade dans la neige, l'omniprésence de ce champ lexical s'explique par sa connotation de froid. Il est manifeste que se dessine ici le motif qui va devenir central dans le second chapitre des Géorgiques, l'omniprésence d'un "froid d'acier ", traité cette fois par Claude Simon sur le mode d'un récit quasi fantastique.

\section{Une évolution stylistique : vers un paysage déréalisé}

La progression dans l'écriture se vérifie non seulement au niveau des détails sensoriels donnés dans chacune des trois sections, mais également au niveau des images utilisées. Alors que les images sont rares dans la première partie, les deux dernières en regorgent. De même, Claude Simon n'utilise les verbes "sembler » et " (ap) paraître " que dans les deux dernières sections. Le trait est aussi plus précis. Ainsi les arbres, qui ne sont pas définis dans la première section, sont identifiés dans les deux dernières comme étant des bouleaux et des pins. Dans ces deux sections, l'écorce des bouleaux est d'ailleurs décrite à l'aide d'une comparaison :

7. Par exemple : «Le ciel est gris, bas, d'une couleur de fer. » (S1, p. 218) ; "Le ciel bas a une couleur métallique légèrement cuivrée. " (S2, p. 218) ; "Le ciel est sombre, décoloré, les masses étirées des nuages immobilisées en une succession de bandes faiblement boursouflées formant un plafond continu, sans une fissure, modelées ton sur ton à la mine de plomb dans un dégradé de gris métalliques, presque noirs. » (S3, p. 219) 
De près l'écorce des bouleaux apparaît pourtant d'un gris argenté, rosé par endroits, parsemée de fentes horizontales et charbonneuses, comme les lèvres de blessures. (S2, p. 218)

Dans la deuxième section, la comparaison suggère la blessure et s'inscrit ainsi dans le champ lexical de la douleur développé dans toute cette partie. Dans la troisième section, les entailles sont assimilées à des "bouches [...] aux lèvres éclatées» (S3, p. 219). Autant d'expressions qui évoquent un contexte de violence, déjà latent à travers les images inspirées du métal.

Mais l'évolution stylistique sensible dans les trois parties de "Progression dans un paysage enneigé " ne repose pas seulement sur le nombre accru des analogies. Alors que les comparaisons font défaut dans la première section, la deuxième contient surtout des comparaisons que nous pouvons qualifier de réalistes. Dans la troisième, par contre, les équivalences proposées créent une déréalisation du paysage. Pour illustrer cette idée, il suffit de relever les occurrences du connecteur « comme si » qui n'apparaît qu'à partir de la deuxième section : la jument lève ses jambes de devant " comme si elle piaffait " (S2, p. 218). La figure que nous rencontrons dans le troisième fragment semble rendre compte d'un effet de lumière plus irréel encore :

Le silence semble absorber aussitôt tous les bruits. Comme si la calotte striée des nuages bas, étroitement soudée à l'horizon de la plaine enneigée, enfermait la forêt, le paysage tout entier, sous un couvercle d'acier qu'aucun son ne parvient à traverser. (S3, p. 220)

C'est dire que les images que Claude Simon esquisse dans la deuxième section de "Progression dans un paysage enneigé " se prolongent dans la troisième. Et l'auteur y ajoute des métaphores, absentes des deux premières sections. Ainsi les bottes du cavalier aux pieds gelés deviennent-elles des " bottes de fer " $(\mathrm{S} 3, \mathrm{p} .219)$ à cause du froid. Avec un jeu de rappel, le narrateur se plaint que ses pieds semblent pris dans des "étriers de froid» (S3, p. 220). Les mains gelées sont décrites comme si "un clou de glace " s'était enfoncé sous les ongles (S3, p. 119). 
La description des impressions sensorielles ainsi que l'utilisation des comparaisons et des métaphores montrent déjà que les trois sections de "Progression dans un paysage enneigé " ne constituent pas le rendu fidèle d'un événement unique mais que les variations le font naitre par approximation. Remarquons que le rythme n'est pas uniforme non plus : alors que la première section est écrite en stac$c a t o$, la ponctuation (surtout les points et les majuscules) scandant le texte, le rythme de la deuxième partie est legato : la ponctuation devient plus faible et les virgules fluidifient le rythme. Dans l'exemple suivant, la différence entre le tempo des deux premières sections est frappante :

Il s'enfonce au petit trot dans le bois. Sans presque qu'il ait à la guider sa monture se faufile entre les troncs noirs des arbres. De temps en temps il est obligé de se pencher sur l'encolure pour éviter une branche basse. (S1, p. 217)

C'est tout juste s'il a besoin de déplacer légèrement sa main pour infléchir la course de la jument qui se dirige au petit trot vers la lisière $\mathrm{du}$ bois, se faufilant entre les troncs des arbres. Sur la blancheur de la neige les troncs semblent d'un noir uniforme. (S2, p. 218)

La troisième section fait continuellement varier le rythme :

Il y a aussi de la neige gelée sur les fûts écailleux des pins, d'un gris mauve, dont les saillies offrent des rugosités où elle s'accroche, recouvrant parfois complètement d'un côté sous sa couche grumeleuse les troncs entre lesquels se faufile le cheval au petit trot à peine guidé par les infimes déplacements de la main. (S3, p. 219)

Après un passage saturé de points et de majuscules, Simon ajoute un extrait au rythme plus fluide, avant de retourner au staccato. Tout comme pour les images utilisées ainsi que certains détails sensoriels, la troisième section reprend les thèmes, figures et rythmes des deux premières.

\section{La variation apportée par Les Géorgiques}

Comme l'écrivait Jean-Yves Laurichesse dans son introduction à " Progression dans un paysage enneigé » dans les Cahiers Claude 
Simon de 2007, ce texte donne à lire une belle phénoménologie de l'hiver qui préfigure sans aucun doute l'" apocalypse de froid " $G$, p. 124) que relate Les Géorgiques. En effet, une analyse approfondie des deux textes simoniens dévoile des liens manifestes entre "Progression » et le roman même.

Dans un premier temps, l'écriture sensorielle qui caractérise le texte court se retrouve dans le roman avec le même type de jeux et d'images. Ainsi la filiation entre "Progression " et Les Géorgiques paraît directe s'agissant du froid. Dans le roman, comme dans le texte court, Simon insiste sur la température glaciale causant des douleurs dans les extrémités du corps du cavalier :

[...] les mains glacées, les doigts glacés, les ongles comme des clous de glace, les pieds glacés dans les chaussures elles-mêmes comme des étaux de glace [...] et ils sont de nouveau au pas, et la glace commence à remonter à partir des pieds le long des jambes, puis ils ne sentent même plus leurs ongles?. ( $G$, p. 90)

Les termes utilisés pour rendre compte de ce froid glacial demeurent presque identiques dans Les Géorgiques, à cette différence près que Claude Simon développe et déploie davantage les images en régime romanesque : le " clou de glace " qui " est enfoncé sous chacun des ongles " (S3, p. 219-220) laisse place à une transformation des ongles en "clous de glace " $(G$, p. 90$)$. De la même façon, le froid personnifié qui remonte le long des jambes du cavalier dans "Progression " est comme chosifié dans le roman jusqu'à devenir " cette espèce de chose invisible, impossible à toucher, et pourtant d'une présence, d'une matière aussi dure que l'acier» ( $G$, p. 108).

Ensuite, le jeu sur le silence et les sons relevé dans " Progression » se retrouve également dans Les Géorgiques, par exemple quand les cavaliers sont surpris par " la chute silencieuse d'un paquet de neige glissant d'une branche " $(G, \mathrm{p} .108)$. Les bruits étouffés par la neige, la " chape de silence " (S3, p. 220) que décrit Claude Simon dans le texte court préfigurent sans aucun doute le "silence irréel »

8. Cahiers Claude Simon, Perpignan, Presses Universitaires de Perpignan, 2007, $\mathrm{n}^{\circ} 3$, p. 11.

9. Notons que le froid persiste dans la nouvelle alors que dans Les Géorgiques il alterne avec davantage de chaleur quand les cavaliers se mettent au galop. 
( $G$, p. 135) qui règne dans Les Géorgiques. Si le roman s'attarde aux mêmes bruits que "Progression ", c'est-à-dire au son des étriers qui cognent contre les sabres d'une part ("cliquetis métalliques» $(G$, p. 91)) et au souffle des chevaux de l'autre, le lecteur des Géorgiques est surtout frappé par l'effet d'étrangeté que suscitent les nombreux oxymores qui juxtaposent des expressions mêlant le bruit et le silence : ainsi la "silencieuse confusion de bruits" ( $G$, p. 90) ou encore les « silencieux craquements de la neige sous les pas" ( $G$, p. 91).

Nous retrouvons enfin dans le roman le contraste entre mouvement et immobilité, développé à l'intérieur des deux dernières sections de "Progression ». Dans Les Géorgiques, Claude Simon évoque la marche des chevaux en 1940 : ils se déplacent de manière ralentie, aérienne :

[...] ces animaux héraldiques qui semblaient se déplacer au rythme d'un trot silencieux et aérien, sans effort apparent, comme s'ils flottaient entre deux eaux, se mouvaient dans un film au ralenti [...]. ( $G$, p. 134)

Observons par ailleurs que la comparaison exprimée par « comme si » est d'ordre irréel, comme dans la troisième partie du texte court.

Qui plus est, Claude Simon réutilise dans Les Géorgiques le champ lexical du métal, qui, comme dans le texte court, se rapporte aussi bien au froid qu'au contexte de la guerre. Ainsi, le ciel de neige est " plombé » $(G$, p. 85) pendant la campagne hivernale des cavaliers dans Les Géorgiques. Sa couleur s'apparente toujours au " gris fer» $(G, \text { p. 102 })^{10}$. Dans le roman, Simon garde également l'idée du goût métallique en lien avec le froid, mais ici dans le contexte du vin gelé dans les bidons des cavaliers. Devenu extrêmement froid, l'alcool prend un goût " âcre ", comme s'il était violet, le froid renforçant encore sa saveur métallique ( $G$, p. 79).

À côté de l'écriture sensorielle, il est possible de voir une " progression " du texte court vers le roman de 1981 au niveau des comparaisons et métaphores. Ainsi Claude Simon brode sur l'image de " couvercle d'acier" (S3, p. 220) développée dans la troisième sec-

10. Nous pouvons étendre la liste des exemples : "métallique immobilité " ( $G$, p. 133), "dur comme acier " ( $G$, p. 108), " des clochers comme des clous dans le ciel d'hiver» $(G$, p. 135), «le clou de glace» $(G$, p. 90$)$. 
tion de "Progression dans un paysage enneigé »: il associe dans Les Géorgiques silence et couche de verre pour souligner l'étrangeté :

Les sons des cloches attachées à leurs cous [des vaches] et les aboiements du chien parvenaient affaiblis et avec un moment de retard, comme s'ils avaient à traverser une épaisse couche de verre, une pellicule à la fois transparente et opaque qui isolerait le visage du monde extérieur. ( $G$, p. 51)

La comparaison interviendra d'ailleurs à nouveau, dans un autre contexte, celui de l'approche de la mort, pour le personnage de LSM, en fin de roman :

[...] comme si pour arriver jusqu'aux tympans du vieil hommemontagne assis sur la terrasse ils [les tintements des cloches] devaient traverser des épaisseurs de silence [...] comme si celui qui allait bientôt mourir était déjà séparé de ce monde aux multiples frémissements, aux minuscules échos, par une vitre, une de ces épaisses plaques de verre qui, dans les aquariums, délimitent les empires de deux éléments [...]. (G, p. 379)

Image qui n'est toutefois pas nouvelle puisque nous la voyons apparaître déjà dans L'Herbe (1958) : " [...] et entre eux deux de nouveau le ciel comme une plaque de verre striée en tous sens par les traits noirs des branches [...] $»^{11}$. Dans Histoire (1967), Claude Simon écrit le passage suivant : « la voix me parvenant comme à travers une plaque de verre, les paroles semblant arriver de très loin, peutêtre parce que le personnage ne paraissait pas tout à fait réel $»^{12}$. Et l'auteur réutilise cette image sous forme de métaphore dans L'Acacia. Le « couvercle d'acier " de "Progression dans un paysage enneigé » y est devenu " une cloche de verre » enserrant le personnage et rendant tous les bruits inaudibles :

[...] comme si tout se déroulait au ralenti, de sorte que plus tard, quand il essaya de raconter ces choses [la longue chevauchée derrière son colonel et deux autres soldats après l'anéantissement de son régiment en mai 1940], il se rend compte qu'il avait fabriqué au lieu de l'informe, de l'invertébré, une relation d'événements telle qu'un esprit normal [...]

11. Claude Simon, L'Herbe, Paris, Minuit, 1958, p. 15.

12. Ibid., p. 49. 
pouvait la constituer après coup [...] tandis qu’à la vérité cela n’avait ni formes définies, ni noms, ni adjectifs, ni sujets, ni compléments, ni ponctuation [...] ni consistance sinon celle, visqueuse, trouble, molle, indécise, de ce qui lui parvenait à travers cette cloche de verre plus ou moins transparente sous laquelle il se trouvait enfermé $[\ldots]^{13}$.

[...] le monde, les choses toujours derrière l'épaisse cloche de sommeil dans le soleil aveuglant, jaune et noir, les formes distendues télescopées par les parois du verre $[\ldots]^{14}$.

Ces exemples ponctuels de phénomènes intratextuels entre "Progression dans un paysage enneigé » et Les Géorgiques ne constituent pas les seuls liens entre les deux textes. Le rythme de " Progression" - staccato puis legato - annonce lui aussi le roman de 1981. La composition des Géorgiques s'inspire directement du texte court : le roman est composé de manière symétrique en cinq parties, mais du premier chapitre au cinquième, le rythme de la phrase alterne des staccato et des legato. La juxtaposition d'une même scène des Géorgiques montre que Simon passe d'un rythme rapide dans le premier chapitre à de longues phrases ininterrompues dans le dernier, de sorte qu'un extrait finit par se superposer à un autre ${ }^{15}$.

Il est donc raisonnable d'accepter que le travail sur le rythme, si caractéristique des Géorgiques, ait déjà préoccupé Claude Simon à l'époque de l'écriture de "Progression dans un paysage enneigé ».

Contrairement à " Progression dans un paysage enneigé ", Les Géorgiques ne raconte pas seulement l'histoire du jeune cavalier pendant la campagne de 1940. Ce livre s'arrête également au personnage plus âgé qui écrit des romans. La main de ce personnage fait son apparition très tôt dans le roman :

Il cesse de feuilleter les cahiers et regarde sa main dans le soleil qui fait ressortir les milliers de rides plus ou moins larges se chevauchant,

13. Claude Simon, L'Acacia, Paris, Minuit, 1989, p. 286-287.

14. Ibid., p. 305-306.

15. Cf. Pierre Schoentjes, Claude Simon par correspondance, Genève, Droz, 1995, coll. «Romanica Gandensia », p. 126-128. Remarquons que la musique sera aussi thématiquement présente dans Les Géorgiques: l'opéra de Gluck auquel assiste le narrateur quand il est jeune. 
s'entrecroisant, mais toutes orientées dans le même sens, comme des plissements de terrain. ( $G$, p. 28)

Simon développe le motif surtout dans le cinquième chapitre, qui focalise sur le cavalier devenu un écrivain âgé. Si aucun personnage d'écrivain ne figure dans "Progression dans un paysage enneigé ", l'attention particulière à la main s'avère déjà présente, mais il s'agit ici de la main du cavalier ${ }^{16}$. Ce n'est sans doute qu'après avoir lu Les Géorgiques que nous comprenons rétrospectivement l'importance de la main dans le texte court. Nous pouvons lier sa présence à la métaphore de l'écriture que développe Claude Simon dans son roman. La mine de plomb, brièvement mentionnée dans " Progression dans un paysage enneigé ${ }^{17}$ et qui réapparaît à plusieurs reprises dans le roman, se rapporte également à la métaphore de l'écriture. Elle représente l'écriture pointue, les détails minutieusement rendus par l'écrivain à l'aide d'" une mine de plomb soigneusement et constamment (de façon presque maniaque) réaffûtée par l'artiste au cours de son travail » $(G$, p. 12). Le mot est d'autant plus intéressant pour Simon qu'il rejoint également le champ lexical du métal, développé tout au long du texte court et du roman.

Hormis la main et la mine de plomb, les nombreuses mentions d'objets de couleur noire sur un arrière-fond blanc évoquent l'écriture à l'encre noire sur une page blanche. Pensons par exemple aux arbres qui parsèment la nature enneigée : "sur la blancheur de la neige les troncs semblent d'un noir uniforme " (S2, p. 218). Le contraste entre le noir et le blanc dans la nature est surtout développée dans la troisième partie du texte, où l'auteur s'attache aux

16. Voici quelques exemples des gestes de la main : "C'est tout juste s'il a besoin de déplacer légèrement sa main pour infléchir la course de la jument qui se dirige au petit trot vers la lisière du bois, se faufilant entre les troncs des arbres. " ( $\mathrm{S} 2$, p. 218); «Il l'aide à prendre appui sur le mors en élevant la main qui tient les rênes et serre les cuisses pour se maintenir en selle.» (S2, p. 218); «[...] les troncs entre lesquels se faufile le cheval au petit trot à peine guidé par les infimes déplacements de la main. " (S3, p. 219).

17. Dans la troisième section, Simon utilise l'image de la mine de plomb pour spécifier la couleur du ciel : « des nuages immobilisées en une succession de bandes faiblement boursouflées [...] modelées ton sur ton à la mine de plomb » (S3, p. 219). 
" extrémités des chaumes qui crèvent la croûte gelée " (S3, p. 221) dont la terre est recouverte. De la même façon, dans Les Géorgiques, ce sont les corbeaux qui créent des taches noires quand ils survolent les champs enneigés :

[...] l'horizon bleuâtre des bois, la campagne enneigée sous le ciel bilieux où tournoyaient sans fin les mêmes vols de corbeaux lançant leurs cris discordants, virant en s'inclinant, s'abattant, pointillant de taches noires la neige sur laquelle semblaient dessinés à la mine de plomb les robustes petits chevaux aux paturons barbus, aux puissantes encolures, secouant leur crinière sauvage, s'ébrouant lorsque passaient les pelotons lancés au grand trot dans le crépitement assourdi des sabots $[\ldots]^{18} .(G$, p. 134).

Mais c'est un vol d'un autre type d'oiseaux sur lequel Claude Simon attire davantage notre attention dans Les Géorgiques, à savoir celui des corneilles. Plusieurs critiques littéraires se sont penchés sur

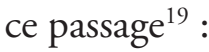

Le vol des corneilles s'éloigne peu à peu. En fait il se décompose en une multitude de vols tournoyants, sans liens apparents, de sorte que l'essaim noir est animé d'un double mouvement : celui qui le déporte lentement et, à l'intérieur, cette quantité de remous, de retours en arrière, de boucles décrites dans des plans verticaux ou obliques donnant l'impression d'un désordre qui n'influe cependant en rien sur le déplacement de l'ensemble. ( $G$, p. 36)

Même si les oiseaux évoluent dans un désordre total à l'intérieur de la volée, celle-ci suggère un mouvement vers l'avant. Le vol des corneilles serait une mise en abyme de l'écriture du romancier : chez Simon, différents motifs resurgissent qui peuvent se croiser et se prolonger. Un fragment compte toujours moins en lui-même qu'en fonction de l'ensemble dans lequel il doit s'insérer ${ }^{20}$. Ce n'est qu'une

18. On trouve d'autres exemples dans Les Géorgiques: «Le givre, la surface ternie des étangs gelés, les vols tournoyants et criards, se répercutant dans le silence, les corbeaux qui s'abattaient dans les champs enneigés. » (G, p. 108).

19. Lire à ce sujet Guy Neumann, "Simon et Michelet : exemple d'intertextualité génératrice dans Les Géorgiques ", Australian Journal of French Studies, 24, 1987, p. 83-99; Pierre Schoentjes, op. cit.

20. Cf. Pierre Schoentjes, op. cit., p. 136. 
fois le texte simonien lu dans son intégralité, que le lecteur en perçoit la cohérence.

À regarder de près, cette lecture des Géorgiques est préfigurée dans « Progression dans un paysage enneigé ». Les oiseaux surgissent brusquement dans la troisième section de ce texte par le biais des traces que laissent leurs pattes dans la neige :

On n'entend pas un seul oiseau dans le bois. [...] Pourtant, tout à l'heure, alors que le cheval peinait, enfoncé jusqu'au poitrail, il a pu voir les empreintes délicates laissées sur la neige par des pattes en forme de trident : un chapelet de minuscules éventails dessinant des arabesques paresseuses, sans but apparent, revenant parfois sur elles-mêmes, se recoupant, traçant des boucles. (S3, p. 220)

Les traces légères des oiseaux répètent à une échelle inférieure celles laissées par le cheval qui s'enfonce jusqu'au poitrail dans la neige. Comme dans le roman, nous remarquons ici une superposition de traces et de traits : la ligne quasiment droite des pas du cheval recouvre les traces mêlées des oiseaux ${ }^{21}$. Cette schématisation rappelle d'ailleurs le dessin stylisé que Simon utilise fréquemment dans ses œuvres ; ainsi déjà dans La Route des Flandres :

[...] de sorte que le trajet suivi par chaque unité aurait pu être schématiquement représenté par une de ces lignes fléchées ou vecteur [...] les collines figurées sur la carte au moyen de petits traits en éventail bordant la ligne onduleuse d'une crête $[\ldots]^{22}$.

21. Observons que cet extrait de "Progression " n'annonce pas uniquement la métaphore du vol des corneilles dans Les Géorgiques mais préfigure également les lettres de LSM réécrites. En effet, le narrateur essaie de lire les phrases maladroites, " avec leurs festons irréguliers de jambages, d'entrelacs, de ratures, d'arabesques, les lignes dessinent sur les feuillets jaunis comme de minces bandes de dentelles déchiquetées et fanées " $(G$, p. 58).

22. Claude Simon, La Route des Flandres, Paris, Minuit, 1984, coll. "Double ", p. 280-281. Dans son roman suivant, Le Palace, nous retrouvons un schéma analogue : « [...] en train de dessiner, [...] vers l'intérieur du rectangle, une courte ligne ondulée, comme un serpent, une sinusoïde, puis, sans explication, au-dessus de celle-ci et à la verticale [...] un cercle ou plutôt plusieurs cercles [...] concentriques, se superposant, ou légèrement excentriques les uns par rapport aux autres, découvrant lorsqu'il écarta la main, quelque chose qui ressemblait à une pelote de laine [...]. »(Claude Simon, Le Palace, Paris, Minuit, 1962, p. 61). 
À l'image des traces laissées par les oiseaux et le cheval, l'écriture simonienne avance petit à petit à travers des fragments qui se touchent, réapparaissent et disparaissent. Une fois la lecture du texte court terminée, nous comprenons qu'il serait possible d'envisager le paysage enneigé dans lequel progresse le cavalier comme une autre métaphore du dessin à la mine de plomb : il représenterait la feuille blanche qui se remplit. Cette métaphore s'impose d'autant plus naturellement que les trois sections du texte se présentent dans un ordre de longueur croissant. La progression dans la neige se transforme ainsi de manière imagée en une progression dans le dessin.

\section{Des impressions de lecture toutefois dissemblables}

Édité pour la première fois en 1976, "Progression dans un paysage enneigé "s'inscrit clairement dans la genèse des Géorgiques, même si l'auteur ne l'a pas intégré littéralement dans la version finale du roman. «Progression » entre en résonance avec le roman de 1981, aussi bien au niveau thématique que stylistique et formel. Le texte court peut donc en quelque sorte être considéré comme une miniature des Géorgiques. Ce roman prolonge un jeu de variations stylistiques, formelles et thématiques que propose déjà « Progression dans un paysage enneigé ". En effet, même si Claude Simon n’a pas réutilisé de manière littérale ces extraits dans son roman ultérieur, nous pouvons facilement repérer les passages des Géorgiques qui portent la marque de cette écriture préalable.

Les fragments épars juxtaposés dans " Progression dans un paysage enneigé » créent une atmosphère qui differe de celle des variations sur ces fragments dans Les Géorgiques, même si la rupture de ton n'est pas très marquée. Cette impression de lecture différente provient surtout du fait que les passages se lisent tels quels dans "Progression ", alors que dans le roman ils entrent en résonnance avec l'histoire de Jean-Pierre Lacombe Saint-Michel. La confrontation entre l'histoire du cavalier pendant la Seconde Guerre mondiale et celle de l'ancien général de la Convention montre à quel point les événements historiques se répètent ${ }^{23}$.

23. Jean-Yves Laurichesse remarque que si Claude Simon avait conservé les frag- 
Les variations dans l'exécution du dessin à la mine de plomb réitèrent les variations intervenant dans l'Histoire. "Progression " n'implique en rien l'idée d'un monde évoluant vers une perfection plus grande. Son progrès est similaire à celui de l'écriture : pour l'Histoire comme pour l'écrivain il s'agit de passer inlassablement par des points identiques où se jouent à chaque fois des variations signifiantes.

ments du texte court, le cavalier solitaire dans "Progression ", cet homme au "vaste manteau dont il a rejeté un pan par-dessus son épaule » $(\mathrm{S} 1, P, 218)$, aurait même pu être identifié comme le général LSM et créer ainsi un lien supplémentaire entre les différents éléments du roman. 